\title{
In situ TEM observation of spontaneous alloying in nanometer-sized particles
}

\author{
H MORI and H YASUDA \\ Research Centre for Ultra-High Voltage Electron Microscopy, Osaka University, Yamadaoka, Suita, \\ Osaka 5650871 , Japan
}

\begin{abstract}
The alloying behaviour in nanometer (nm)-sized particles was studied by transmission electron microscopy (TEM). When solute atoms are vapour-deposited onto nm-sized particles at room temperature, rapid dissolution of solute atoms into particles occur, and solid solution or compound particles are successfully formed. Such spontaneous alloying occurs even between $\mathrm{nm}$-sized particles of different elements. Our results can be summarized as: (i) spontaneous alloying takes place via a solid-state process, (ii) spontaneous alloying becomes more difficult with increasing particle size, (iii) spontaneous alloying is not an artifact originating from the temperature rise in particles which might be induced by heat of condensation and (iv) remarkable enhancement of solubility is observed in nm-sized compound particles.
\end{abstract}

Keywords. Nanometer-sized particle; spontaneous alloying; phase stability; transmission electron microscopy.

\section{Introduction}

Interest in the studies of ultra-fine particles in the size range from a few to several nanometers $(\mathrm{nm})$ has rapidly been growing in recent years, since the nm-sized particles often exhibit properties and structures which are quite different from those of the corresponding bulk materials (Andres et al 1989). For example, drastic changes in properties such as electronic, optical, and photocatalysis have been found as the size of the system reduces (Halperin 1986). Recently it was found that alloying in nm-sized particles took place at an extremely high rate compared to the rate of interdiffusion in bulk materials (Mori et al 1991; Yasuda and Mori 1992). For example, it was revealed that instantaneous dissolution of copper atoms occurs in nm-sized gold particles at room temperature or reduced temperatures, resulting in successful formation of solid solution particles (Yasuda et al 1992). Subsequently, it was also confirmed that particles of compounds such as $\mathrm{AuSb}_{2}$, InSb, and $\mathrm{AlSb}$ can be produced via similar rapid spontaneous alloying (Mori and Yasuda 1993; Yasuda et al 1994; Yasuda and Mori 1996).

In the present study, dominant factors that govern the process of spontaneous alloying were studied by transmission electron microscopy (TEM) namely, (i) the alloying behaviour of copper atoms into gold particles was investigated in order to get an insight into the atomistic process involved in spontaneous alloying; (ii) the alloying between gold and antimony particles was investigated in an attempt to see whether the heat of condensation of solute atoms is essential in spontaneous alloying; and (iii) the phase stability in $\mathrm{AuSb}_{2}$ compound particles was studied by analytical electron microscopy.

\section{Experimental}

Preparation of gold particles and subsequent vapourdeposition of copper atoms onto the gold particles were carried out using a double-source evaporator in an electron microscope. An amorphous carbon film was used as the supporting film and was mounted on a molybdenum grid.

Using the evaporator, gold was first evaporated to produce nm-sized gold particles on a supporting film. Next, copper atoms were evaporated onto the same film at room temperature. Changes in the morphology and structure of gold particles associated with deposition of the copper atoms were examined by high resolution images (HRIs), bright field images (BFIs), and selected area electron diffraction patterns (SAEDs).

In the second part of experiment, antimony particles on which gold particles were present were prepared in the following manner. Antimony was first evaporated to produce $\mathrm{nm}$-sized antimony particles on a supporting film. The resulting nm-sized antimony particles showed amorphous structure. The amorphous antimony $(a-\mathrm{Sb})$ particles as well as the supporting film were subsequently cooled down to approximately $96 \mathrm{~K}$. Gold was then evaporated onto the same film to produce gold particles which were located on the free surface of individual $a-\mathrm{Sb}$ particles. Changes in the morphology and structure of $a-\mathrm{Sb}$ particles associated with subsequent annealing were studied in situ by BFIs and SAEDs. The flux of these depositing atoms was of the order of $10^{17} \sim 10^{18} \mathrm{~m}^{-2} \cdot \mathrm{s}^{-1}$. The microscopes used were Hitachi $\mathrm{H}-800$ and $\mathrm{H}-9000$, which operated at an accelerating voltage of $200 \mathrm{kV}$ and $300 \mathrm{kV}$, respectively. The base pressure in the specimen chamber was kept below $5 \times 10^{-5} \mathrm{~Pa}$. 
In the third part of experiment, the phase stability of alloy particles in the Au-Sb system was examined using a $200 \mathrm{kV}$ high resolution electron microscope that was equipped with a field emission gun (Hitachi HF-2000). The chemical composition of individual particles on the film was analyzed by energy dispersive $\mathrm{X}$-ray spectroscopy (EDS). The analyses were carried out using an electron probe of $\sim 1 \mathrm{~nm}$ in diameter. The characteristic $X$-rays of gold and antimony were collected with an ultrathin window $\mathrm{X}$-ray detector at a high take-off angle of $68^{\circ}$. The background was simulated by curve fitting, and was then subtracted. The chemical composition of particles was calculated from the intensity ratio between peaks of characteristic X-rays, using sensitivity factors.

\section{Results}

\subsection{Spontaneous alloying of copper atoms into gold particles at room temperature}

A typical example of spontaneous alloying of copper atoms into gold particles kept at room temperature is shown in figure 1 . Figures $1 \mathrm{a}$ and $\mathrm{a}^{\prime}$ show respectively a low magnification HRI of gold particles formed on a supporting film, and the corresponding SAED. The mean diameter of gold particles is $\sim 6 \mathrm{~nm}$. The Debye rings

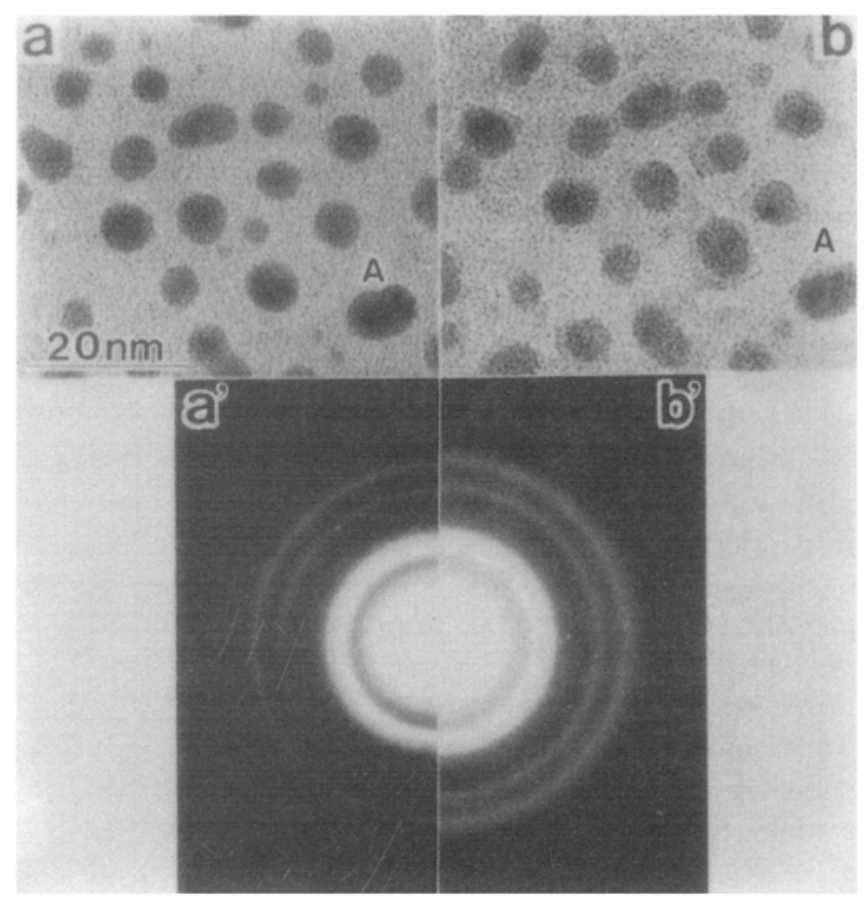

Figure 1. An example of spontaneous alloying of copper atoms into gold particles at room temperature. (a) HRI of gold particles on a supporting film, and $\left(\mathbf{a}^{\prime}\right)$ the corresponding SAED. (b) HRI of the same particles after copper deposition, and $\left(\mathbf{b}^{\prime}\right)$ the corresponding SAED. in the SAED are consistently indexed as those of fcc gold (the lattice constant of gold at room temperature is $0 \cdot 40_{8} \mathrm{~nm}$ ). Figures $1 \mathrm{~b}$ and $\mathrm{b}^{\prime}$ show respectively the same area as in figure 1a after copper deposition, and the corresponding SAED. All the Debye rings in figure $1 b^{\prime}$ can be consistently indexed as those of an fcc crystal with a lattice constant of $0.39, \mathrm{~nm}$. This rapid shrinkage in the lattice constant indicates the rapid dissolution of copper into gold particles resulting in the formation of solid solution particles.

Figure $2 \mathrm{a}$ depicts an HRI of gold particle $\mathrm{A}$ in figure 1a. From the image it is evident that the particle is a multiply-twinned crystal showing nearly five-fold orientation. Further, the particle is composed of five twins and the [110] direction, which is common to all five twins, is nearly parallel to the electron beam. The twinned structure in the particle is schematically illustrated in figure 2c. The almost vertical lattice fringes, which appear in twins $\mathrm{X}$ and $\mathrm{Y}$ in figure $2 \mathrm{a}$ are the (111) lattice fringes. An HRI of the particle after copper deposition is depicted in figure $2 b$. It is evident from figures $2 a$ and $b$ that with the deposition and subsequent dissolution of copper no changes were induced in the twinned structure of the particle: the particle remained composed of five twins and retained nearly five-fold orientation.

If it is postulated that a melting process is responsible for the spontaneous alloying, the arrangement and orientation of grains in a particle would be different between conditions before and after copper deposition. However, the present experimental results do not support this postulate; since with copper deposition no changes were induced in the arrangement and orientation of grains in the particle. Thus this observation provides evidence for the process of spontaneous occurring via a purely solidstate process rather than via melting process (Ajayan and Marks 1989a, b).

Figures $3 a$ and $a^{\prime}$ show respectively an example of as-produced gold particles on a supporting film, and the corresponding SAED. The mean size of gold particles is $\sim 30 \mathrm{~nm}$. A BFI of the particles after copper deposition, and the corresponding SAED are depicted in figures $3 b$ and $\mathbf{b}^{\prime}$, respectively. In the SAED all the Debye rings can be indexed as those of fcc gold and copper. In figure $3 \mathrm{~b}$ appearance of Moir patterns in some of the particles can be observed, suggesting thereby that a film of copper is present on gold particles. Thus in gold particles, approximately $30 \mathrm{~nm}$ in the mean size, such rapid spontaneous alloying does not take place, as was observed in $6 \mathrm{~nm}$-sized gold particles (figures 1 and 2), and only a two-phase mixture of pure gold and copper is produced on a supporting film by the depositing copper. This formation of the unalloyed, two-phase mixture is consistent with that predicted by the copper-atomdiffusivity in bulk gold. Thus from these experiments it 


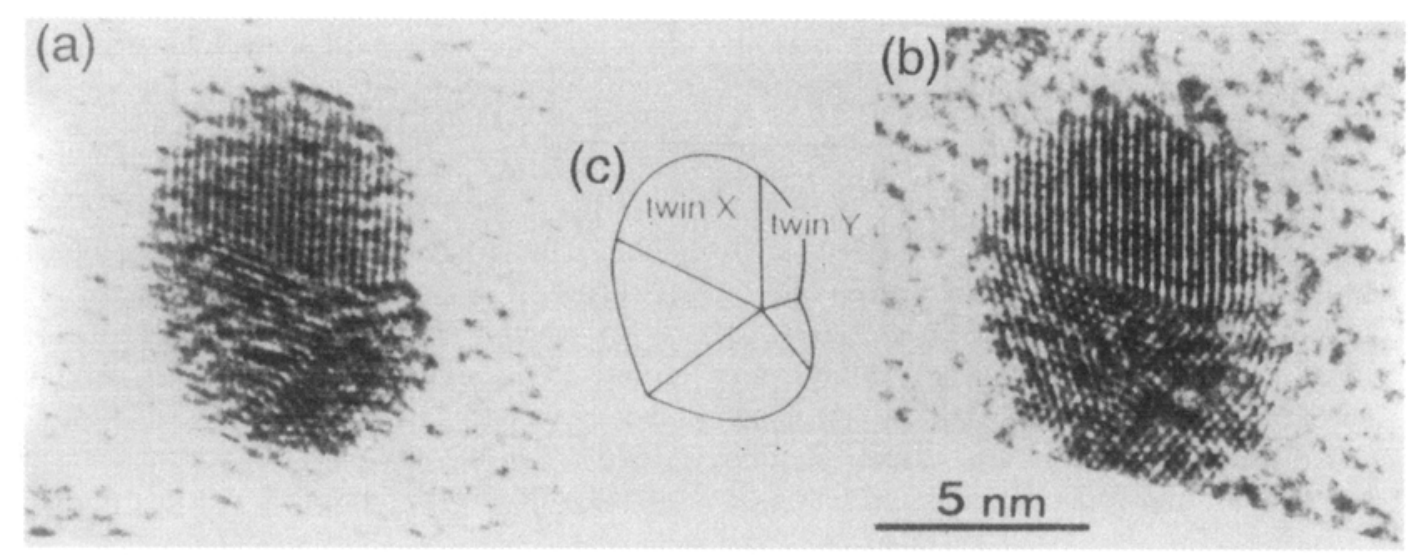

Figure 2. HRIs of particle A (figure 1a) obtained before copper deposition, (b) obtained after copper deposition and (c) a schematic illustration of twin boundaries in particle $A$.

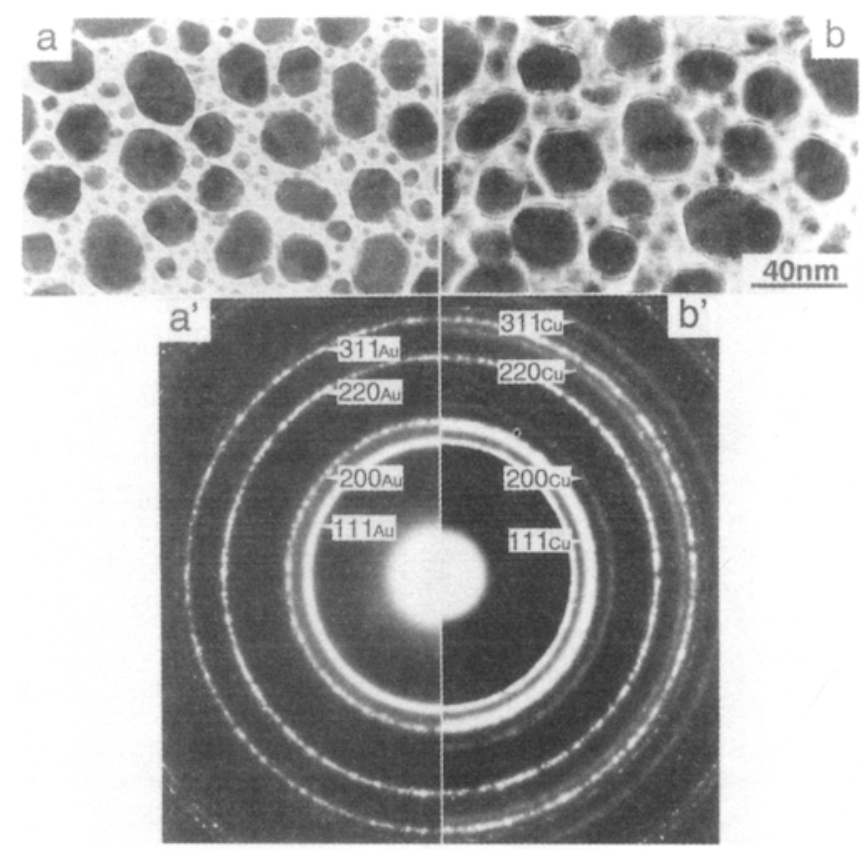

Figure 3. (a) BFI of as-produced $30 \mathrm{~nm}$-sized gold particles, (b) BFI of particles after copper deposition; $\left(\mathbf{a}^{\prime}\right)$ and $\left(\mathbf{b}^{\prime}\right)$ are SAEDs corresponding to (a) and (b), respectively.

is evident that rapid spontaneous alloying becomes more difficult with the increase in particle size.

\subsection{Spontaneous alloying between gold and antimony particles at reduced temperatures}

A typical sequence of spontaneous alloying between gold and antimony particles associated with annealing is shown in figure 4. Figures $4 a$ and $a^{\prime}$ show respectively a BFI of antimony particles after gold deposition at $96 \mathrm{~K}$, and the corresponding SAED. In figure 4a appearance of six antimony particles can be observed, the diameters of which range from 15 to $30 \mathrm{~nm}$. The diameters of gold particles on individual antimony particles range from - 1-4 nm. Particles which are dispersed rather uniformly on the supporting film are gold particles, which we have not considered in the present work. In the SAED (figure $4 a^{\prime}$ ) only Debye rings of fcc gold are recognized, superimposed on halos of $a$-Sb. The value of the scattering vector, $K(=4 \pi \sin \theta / \lambda)$, for the first halo is $\sim 20.5 \mathrm{~nm}^{-1}$. This observation suggests that gold particles formed on $a$-Sb particles at $96 \mathrm{~K}$ remain nonreacted with $a$-Sb particles. Figures $4 \mathrm{~b}$ and $\mathrm{b}^{\prime}$ show respectively a BFI of the same particles after warming up to $250 \mathrm{~K}$, and the corresponding SAED. A comparison of figure $4 \mathrm{a}$ with figure $4 \mathrm{~b}$ shows that at this stage of annealing gold particles (compare such gold particles as Ia to IIIa in figure $4 \mathrm{a}$ with the same particles labelled Ib to IIIb in figure $4 \mathrm{~b}$ ) on $a-\mathrm{Sb}$ particles have decreased in size. In the SAED (figure $4 b^{\prime}$ ) once again only Debye rings of fic gold are recognized, superimposed on halos. These results suggest that dissolution of gold into $a$-Sb particles has been induced at temperatures between 96 and $250 \mathrm{~K}$, although the alloy particles formed remain amorphous. Figures $4 c$ and $c^{\prime}$ show respectively a BFI of the same particles after warming to $270 \mathrm{~K}$, and the corresponding SAED. All the gold particles on alloy particles have disappeared. In the SAED (figure $4 c^{\prime}$ ), Debye rings of $\mathrm{AuSb}_{2}$, albeit significantly interrupted, have appeared, superimposed on Debye rings of $\mathrm{fcc}$ gold and halos from amorphous $\mathrm{Sb}-\mathrm{Au}(\mathrm{a}-(\mathrm{Sb}-\mathrm{Au}))$ alloy particles are present. These observations suggest that the gold concentration in some of $a$-(Sb-Au) alloy particles has now become high enough to attain the stoichiometric composition of $\mathrm{AuSb}_{2}$, and that these $a-(\mathrm{Sb}-\mathrm{Au})$ alloy particles have crystallized into $\mathrm{AuSb}_{2}$ compound particles. Figures $4 \mathrm{~d}$ and $\mathrm{d}^{\prime}$ show respectively a BFI of the same particles after warming up to $290 \mathrm{~K}$, and the corresponding SAED. In the SAED (figure $4 \mathrm{~d}^{\prime}$ ) well-developed Debye rings of $\mathrm{AuSb}_{2}$ are recognized, superimposed on Debye rings of fcc gold. No halos from $a$-(Sb-Au) alloy 
particles are present. This observation suggests that all the $a-(\mathrm{Sb}-\mathrm{Au})$ alloy particles have crystallized into $\mathrm{AuSb}_{2}$ compound particles at $290 \mathrm{~K}$.

Figure 5 indicates a schematic illustration of spontaneous alloying between gold and antimony particles. When gold particles in contact with nm-sized $a$-Sb particles (figure 5a) are gradually heated from 96 to $250 \mathrm{~K}$, dissolution of gold into $a$-Sb particles sets in at temperatures below $250 \mathrm{~K}$ and $a-(\mathrm{Sb}-\mathrm{Au})$ alloys are produced (figure $5 \mathrm{~b}$ ). With the increase in annealing temperature, more gold particles are dissolved into individual $a$-Sb particles, and when the gold concentration in $a-(\mathrm{Sb}-\mathrm{Au})$ alloy particles reaches to the stoichiometric composition of $\mathrm{AuSb}_{2}$, these amorphous particles crystallize into $\mathrm{AuSb}_{2}$ compound particles around 270-290 K (figure $5 \mathrm{c}$ ).

In the case of spontaneous alloying in a system where solute atoms have been supplied as isolated atoms onto particles of a pure substance, there is always a doubt as to whether it could be the heat of condensation of solute atoms which increases the temperature of particles and thus result in an accelerated diffusion. Our present study rules out this possibility, since the heat has been already dissipated during the deposition process (i.e.

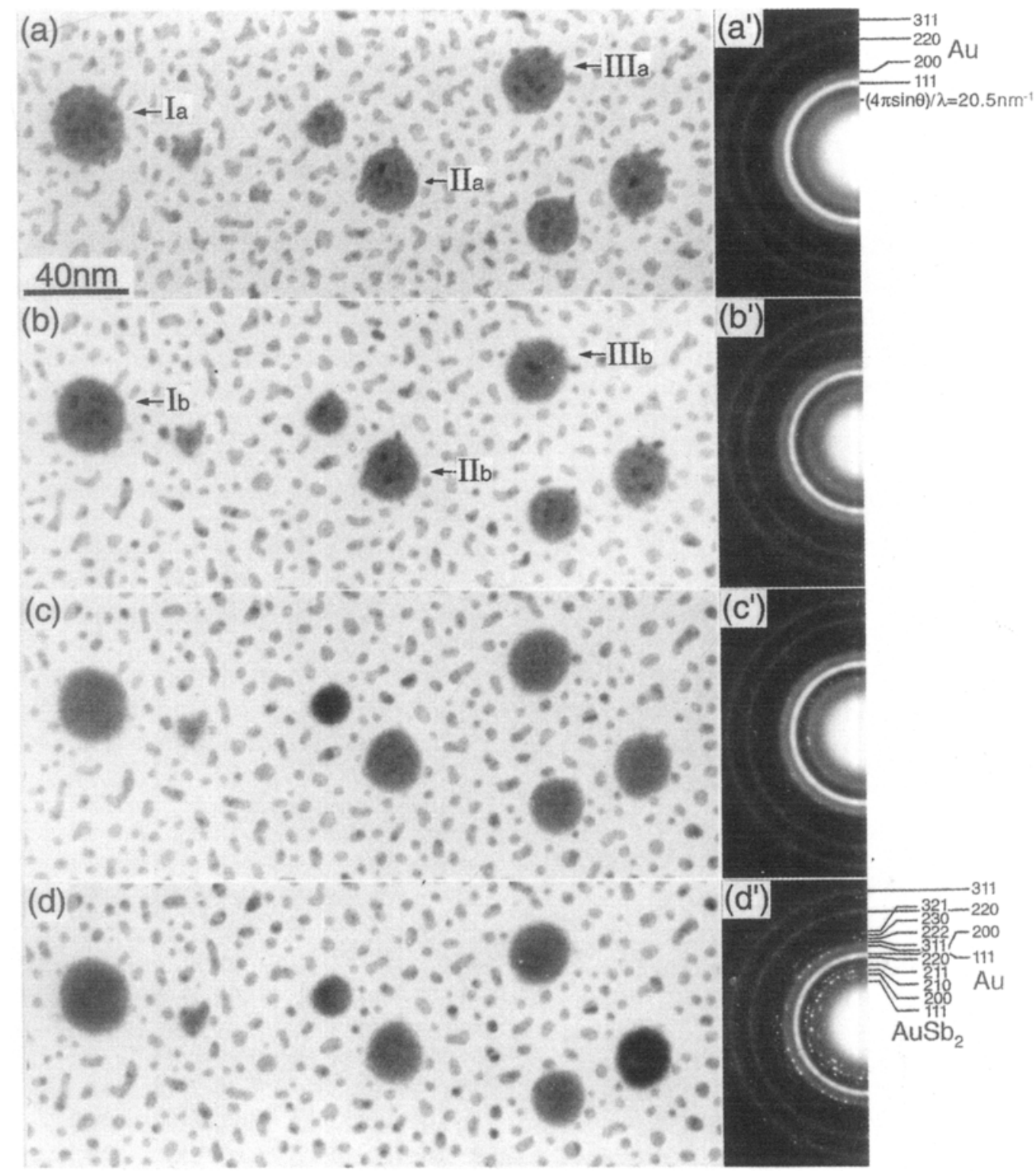

Figure 4. A typical sequence of spontaneous alloying of gold into $a$-Sb particles associated with subsequent annealing. (a) BFI of $a$-Sb particles after gold deposition at $96 \mathrm{~K}$, and ( $\mathbf{a}^{\prime}$ ) the corresponding SAED. (b) BFI of the same particles after warming up to $250 \mathrm{~K}$, and ( $\mathbf{b}^{\prime}$ ) the corresponding SAED. (c) BFI of the same particles after warming up to $270 \mathrm{~K}$, and ( $\mathbf{c}^{\prime}$ ) the corresponding SAED. (d) BFI of the same particles after warming up to $290 \mathrm{~K}$, and (d') the corresponding SAED. 
during the gold deposition at $96 \mathrm{~K}$ in the present case). Thus, the heat of condensation and any temperature rise associated with it plays no essential role in the spontaneous alloying.
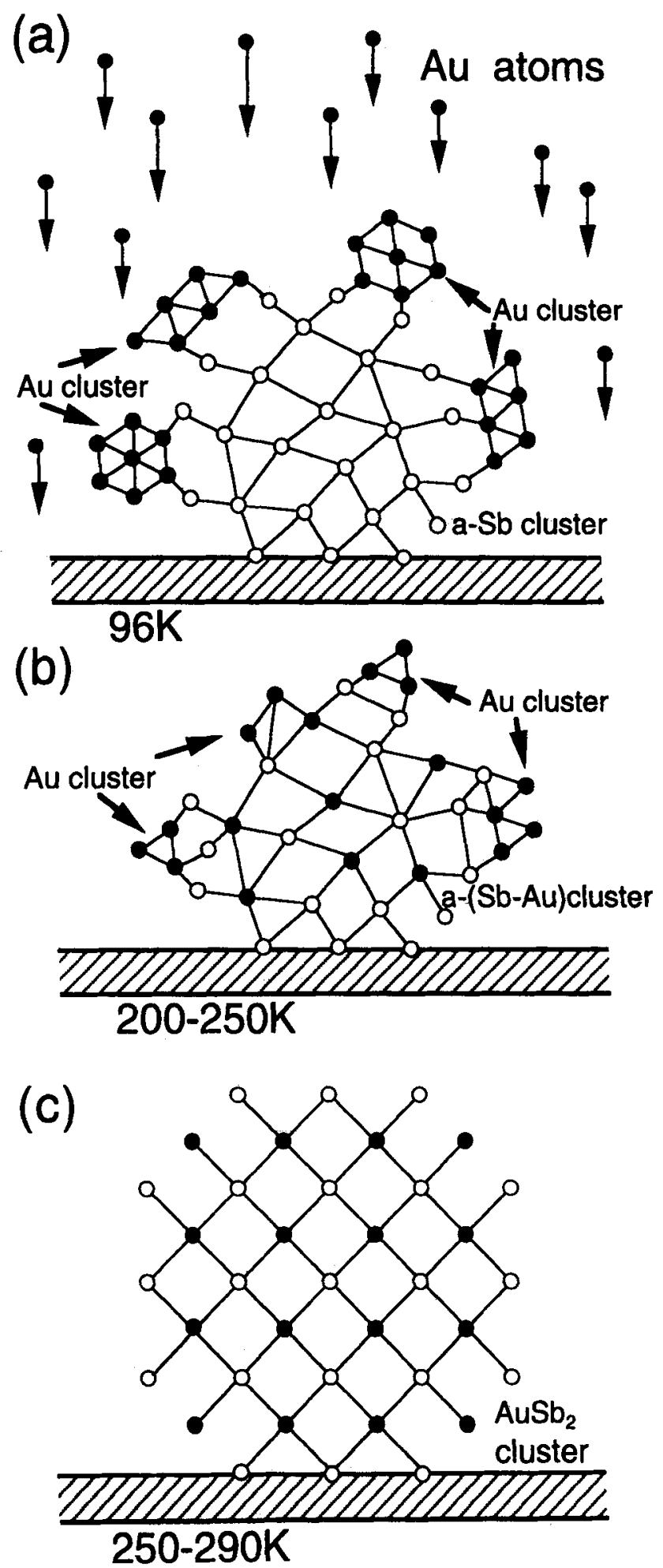

Figure 5. The schematic illustration of spontaneous alloying between gold and antimony particles.

\subsection{Phase stability in $\mathrm{AuSb}{ }_{2}$ compound particles}

Figure 6 shows a series of HRIs of $\mathrm{AuSb}_{2}$ particles with different chemical compositions. The particles were produced by spontaneous alloying. An EDS spectrum taken from the region encircled is shown below each HRI. All the compound particles are single crystalline. The $0.24 \mathrm{~nm}$-spaced fringes in the images are the (220) lattice fringes of $\mathrm{AuSb}_{2}$. These fringes are at an angle of $60^{\circ}$ to each other, showing that the incident beam direction is along the [111] of $\mathrm{AuSb}_{2}$ with the C2 structure. EDS spectra indicate that antimony concentrations of particles shown in figures $6 \mathrm{a}, \mathrm{b}$ and $\mathrm{c}$ respectively are $52 \mathrm{at} \% \mathrm{Sb}$ (which is by $\sim 14$ at\% lower than the stoichiometric composition of $\mathrm{AuSb}_{2}$ ), 65 at\% $\%$ (which is almost equal to the stoichiometric composition of $\mathrm{AuSb}_{2}$ (i.e. 1/3)) and 72 at $\% \mathrm{Sb}$ (which is by $\sim 6$ at\% higher than the stoichiometric composition of $\mathrm{AuSb}_{2}$ ). Thus these experiments confirm that remarkable enhancement of solubility has been induced in $\mathrm{AuSb}_{2}$ compound particles compared with that in the corresponding bulk $\mathrm{AuSb}_{2}$.

\section{Discussion}

\subsection{Temperature rise of particles during in situ} observation of spontaneous alloying

A brief discussion follows to consider whether spontaneous alloying in the nm-sized particles could be an artifact that originates due to temperature rise in the particles. Three major causes for the temperature rise are electron beam heating, heat of condensation, and heat of mixing. Our previous work by UV-visible absorption spectroscopy (Yasuda and Mori 1997) has revealed that electron illumination is not a prerequisite to the spontaneous alloying in nm-sized particles. In the literature it has been reported that the cohesive energy of copper is $336 \mathrm{~kJ} \cdot \mathrm{mol}^{-1}$ (Kittel 1956) and that the heat of mixing for an $\mathrm{Au}_{0.5} \mathrm{Cu}_{0.5}$ alloy at $300 \mathrm{~K}$ is $8.75 \mathrm{~kJ} \cdot \mathrm{mol}^{-1}$ (Hultgren et al 1973). It is considered from these values that the temperature rise in particles due to the heat of mixing is $\sim 2$ orders of magnitude lower than that due to the heat of condensation. In the present work, it has been confirmed that the heat of condensation and any temperature rise associated with it plays no essential role in the spontaneous alloying. Thus, spontaneous alloying in nm-sized particles is not an artifact originating from the temperature rise in particles, but rather is an intrinsic property of $\mathrm{nm}$-sized particles.

\subsection{Generality in spontaneous alloying}

It has been confirmed in our previous experiments that solid solution or compound particles are formed by 


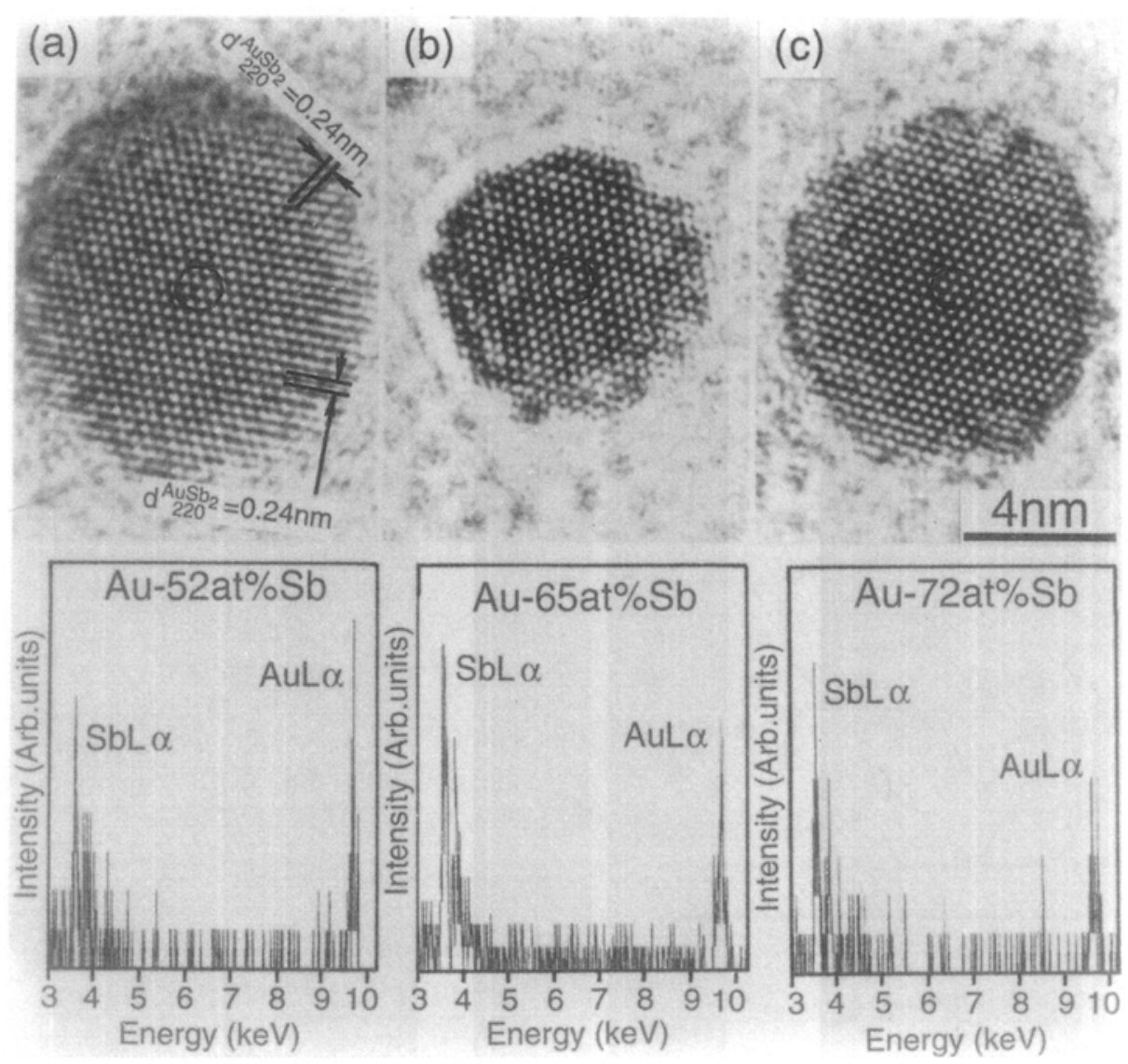

Figure 6. HRIs of $\mathrm{AuSb}_{2}$ compound particles with different antimony concentrations. EDS spectrum taken from the region encircled in the image is shown below each micrograph.

spontaneous alloying in a lot of binary systems. In the $\mathrm{Au}-\mathrm{Cu}$ (Yasuda and Mori 1994), Au-Zn (Yasuda and Mori 1992) and Au-Al (Mori and Yasuda 1995) systems in which the heat of formation is negative, spontaneous alloying takes place with ease. In the $\mathrm{Au}-\mathrm{Ni}$ system wherein the heat of formation is slightly positive, spontaneous alloying takes place but the critical size becomes quite small. On the other hand in the In-Al system where the heat of formation is largely positive, no spontaneous alloying occurs. Thus in nm-sized particles, atom mixing driven by the difference in chemical free energy could be induced with ease.

\subsection{Feature of phase stability in compound particles}

It has been confirmed that the compound particles tend to remain in a single phase, even if the composition of compound particles falls in the two-phase region in the equilibrium phase diagram of bulk materials. In compound particles the lattice distortion, which might be induced by the deviation from the stoichiometric composition, could be relaxed rather easily than in bulk material, because the lattice itself becomes elastically soft to some extent (Matsubara et al 1977; Harada and Ohshima 1981). It is considered that the increase of solid solubility observed here may be an intrinsic feature of the phase stability in nm-sized compound particles. Systematic studies on the structure and chemical composition of $\mathrm{nm}$-sized alloy particles are in progress in our laboratory.

\section{Conclusion}

The alloying behaviour in $\mathrm{nm}$-sized particles has been studied in situ by TEM. When solute atoms are vapourdeposited onto nm-sized particles at room temperature, rapid dissolution of solute atoms into particles takes place, and solid solution or compound particles are successfully formed. Such spontaneous alloying occurs even between nm-sized particles of different elements. Results obtained can be summarized as: (i) spontaneous alloying takes place via a solid-state process, (ii) spontaneous alloying becomes more difficult to take place with increasing particle size, (iii) spontaneous alloying 
is not an artifact originating from the temperature rise in particles which might be induced by heat of condensation and (iv) remarkable enhancement of solubility is observed in $\mathrm{nm}$-sized compound particles.

\section{Acknowledgements}

This work is, in part, supported by the programme, 'Research for the Future', JSPS \#96P00305, and the Ministry of Education, Science, Sports and Culture under Grant-in-Aid for Scientific Research.

\section{References}

Ajayan P M and Marks L D 1989a Phys. Rev. Lett. 60585 Ajayan P M and Marks L D 1989b Phys. Rev. Lett. 63279 Andres R P et al 1989 J. Mater. Sci. 4704 Halperin W P 1986 Rev. Mod. Phys. 58533
Harada J and Ohshima K 1981 Surf. Sci. 10651

Hultgren R, Desai P D, Hawkins D T, Gleiser M and Kelley K K 1973 Selected values of the thermodynamic properties of binary alloys (Ohio: American Society for Metals) p. 332

Kittel C 1956 Introduction to solid state physics (New York: John Wiley) 2nd ed. p. 99

Matsubara T, Iwase Y and Momokita A 1977 Prog. Theor. Phys. $58 \quad 1102$

Mori H and Yasuda H 1993 Intermetallics 135

Mori H and Yasuda H $1995 \mathrm{~J}$. Microsc. 18033

Mori H, Komatsu M, Takeda K and Fujita H 1991 Philos. Mag. Lett. 63173

Yasuda H and Mori H 1992 Phys. Rev. Lett. 693747

Yasuda H and Mori H 1994 Z. Phys. D31 131

Yasuda H and Mori H 1996 Mater. Sci. Eng. A217/218 249

Yasuda H and Mori H 1997 Z. Phys. D40 144

Yasuda H, Mori H, Komatsu M and Takeda K 1992 Appl. Phys. Lett. 612173

Yasuda H, Mori H, Muraki T and Sakata T 1994 Z. Phys. D31 209 